\title{
Accelerating Voltage and Probe Current Dependence of Electron Beam Drilling Rates for Silicon Crystal
}

\author{
Noriaki Endo ${ }^{1}$ and Yukihito Kondo ${ }^{1}$ \\ 1. JEOL Ltd., 3-1-2 Musashino, Akishima, Tokyo, Japan.
}

Energy dispersive X-ray spectroscopy (EDS) and/or electron energy-loss spectroscopy (EELS) in scanning transmission electron microscopy (STEM) is very popular for analysis of semiconductor devices, since we require chemical information of them other than their shapes and dimensions. However, it requires more electron dose onto a sample than that of imaging due to small signal intensity. It is unavoidable that the sample lamella suffers the electron beam damage from electrons of an STEM probe in EDS and/or EELS analysis. The damage could be categorized into two types, one is structure deformation of sample and the other is beam drilling caused from etching and/or migration of sample atoms [1]. The latter is crucial for elemental analysis, since it significantly affect to results of compositional and/or quantitative chemical analyses. Several results on the beam drilling effect have been reported so far [2, 3]. In this paper, we report beam drilling of Si crystal which depends on accelerating voltage and electron probe current using EDS, because it could be a crucial problem and should be avoided for long time or high magnification analysis.

We used a field emission electron microscope (JEOL, JEM-2800) equipped with Schottky FEG and two large-sized silicon drift detectors (dual SDD, $100 \mathrm{~mm}^{2}$ ). A lamella of a silicon device $(\operatorname{Si}(110)$ ) was prepared by $\mathrm{Ar}^{+}$ion milling (JEOL, Ion Slicer). The thickness of the lamella was measured to be approximately $15 \mathrm{~nm}$ by the EELS ratio method. To estimate the electron beam drilling rate, we measured a decay of Si X-ray $(\mathrm{K} \alpha)$ count rate using a point analysis mode.

Figure 1 shows decay profiles of Si count rates for various accelerating voltages. Decay rate becomes slower in the lower accelerating voltages. The measured X-ray count rate $(\boldsymbol{R})$ is thought to be proportional to number of atoms $(\boldsymbol{n})$ in a beam. Therefore, the decay rates are fit with the relation:

$$
\boldsymbol{R}=\boldsymbol{R}_{\boldsymbol{0}} \exp (-\boldsymbol{a t}),
$$

where $\boldsymbol{R}_{\boldsymbol{0}}$ is the initial count rate, $\boldsymbol{a}$ is drilling coefficient at a probe current and an accelerating voltage, and $\boldsymbol{t}$ is elapsed time. $\boldsymbol{n}(\boldsymbol{t})$ is defined under the relation:

$$
d n / d t=n a,
$$

where $\boldsymbol{d n} / \boldsymbol{d} \boldsymbol{t}$ is a sputtering rate of atoms and is proportional to number of atoms in the beam $(\boldsymbol{n})$ and drilling coefficient $(\boldsymbol{a})$. The dependence of the drilling coefficient on relative accelerating voltage is plotted in Figure 2. The drilling rates are not proportional to accelerating voltage. The dependence of the drilling rate on accelerating voltage is related to the threshold energy of sputtering of Si atoms. The drilling rate is very small at $60 \mathrm{kV}$ or less.

Figure 3 shows the decay profiles of Si count rates for various probe currents at $200 \mathrm{kV}$. By reducing a probe current, the drilling rate can be controlled. For analysis of fragile samples, it is better to use small probe current. Figure 4 plots the probe current dependence of the drilling coefficient defined in the above column. The measured drilling rates are approximately proportional to probe current, though the electron densities under these probes are approximately constant to be $2.0 \mathrm{nA} / \mathrm{nm}^{2}$. The reason for this may be related to the migration of atoms to fill the generated hole. And it may be promoted by concerted effect by neighboring irradiating electrons. 
In conclusion, we found out that elemental analysis in the low accelerating voltage is very effective for reduction of the electron beam damage as well as higher sensitivity due to larger ionization cross section of an element. In order to reduce sample damage it is necessary to pay attention to the probe current in the analysis at higher voltages $\geq 200 \mathrm{kV}$.

\section{References:}

[1] RF Egerton, P Li and M Malac, Micron 35 (2004) p.399.

[2] LE Thomas, Ultramicroscopy 18 (1985) p.173.

[3] PA Crozier, MR McCartney and DJ Smith, Sur. Sci. 237 (1990) p.232.

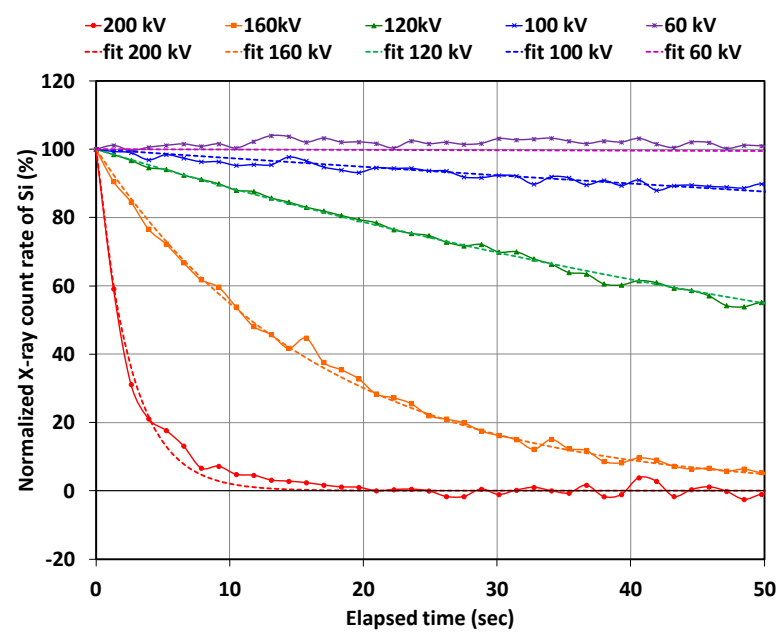

Figure 1. Decay profiles of Si count rates for various acceleration voltages with a probe current of $3.72 \mathrm{nA}$. These decay profiles are

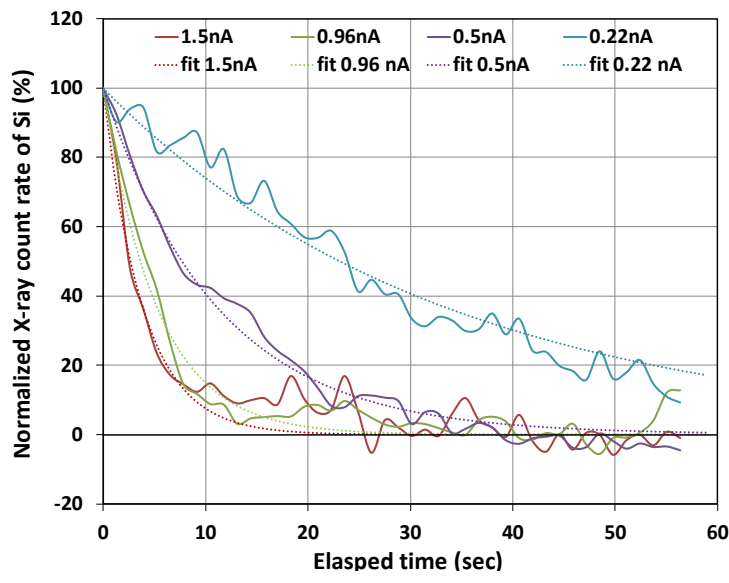

Figure 3. Decay profiles of Si count rates for various probe currents at $200 \mathrm{kV}$. These decay profiles are normalized by initial count rate.

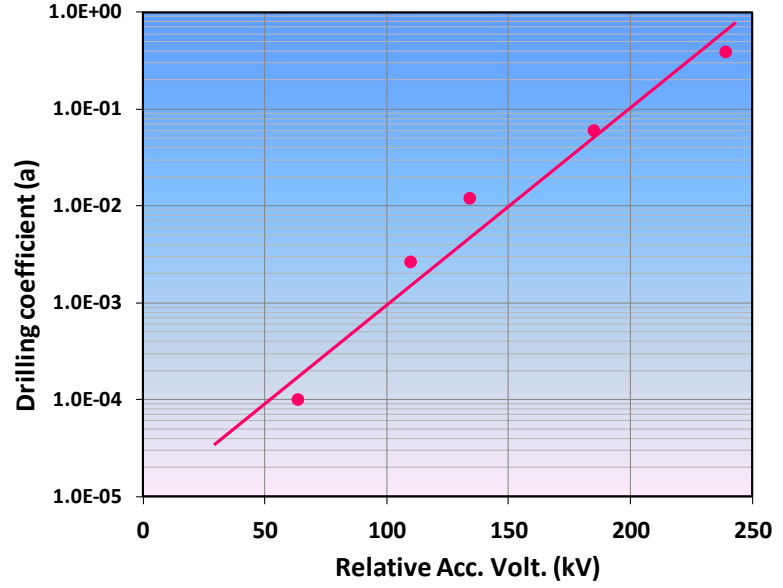

Figure 2. Dependence of drilling coefficient on relative accelerating voltage. The vertical axis scale is logarithmic.

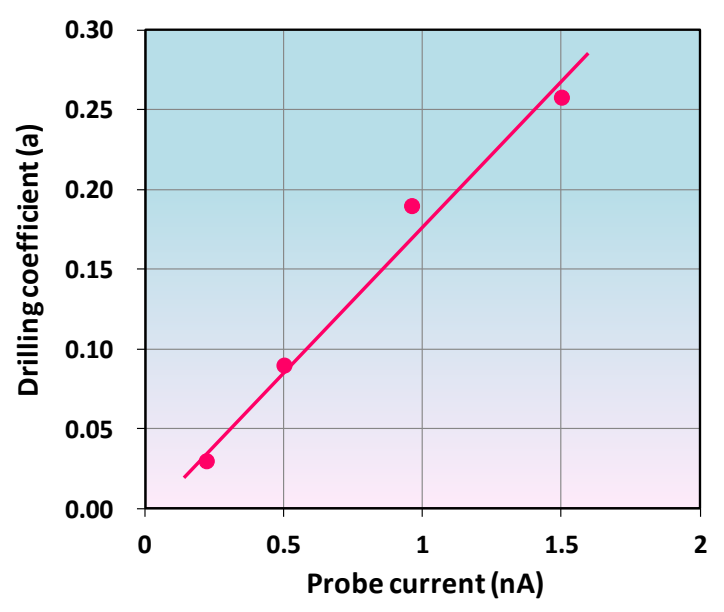

Figure 4. Dependence of drilling coefficient on probe current. The vertical axis scale is linear. 